\title{
A configurable model of the
}

2

\section{synaptic proteome reveals the}

3

\section{molecular mechanisms of disease}

4

\section{co-morbidity.}

5

6

7

8

9

10

11

12

13

14

15

Oksana Sorokina*1, Colin Mclean*1, Mike DR Croning ${ }^{2}$, Katharina F Heil ${ }^{1,4}$, Emilia

Wysocka $^{1}$, Xin $\mathrm{He}^{5}$, David Sterratt ${ }^{1}$, Seth GN Grant ${ }^{2,5}$, T Ian Simpson ${ }^{1,5}$, and J

Douglas Armstrong ${ }^{1,3,5}$.

\section{(1)}

(1)

Email: Colin Mclean* - Colin.D.Mclean@ed.ac.uk and Oksana Sorokina* -

\section{Oksana.Sorokina@ed.ac.uk}

*Corresponding author

1. The School of Informatics, University of Edinburgh, Edinburgh, EH8 9AB,

\section{Scotland.}

2. Centre for Clinical Brain Sciences, University of Edinburgh, Edinburgh EH16 4SB, United Kingdom

3. Computational Biomedicine Institute (IAS-5 / INM-9), Forschungszentrum Jülich, Jülich, Germany

4. University of Barcelona, Barcelona, Spain

5. Simons Initiative for the Developing Brain 
Synapses contain highly complex proteomes which control synaptic transmission, cognition and behaviour. Genes encoding synaptic proteins are associated with neuronal disorders many of which show clinical co-morbidity. Our hypothesis is that there is mechanistic overlap that is emergent from the network properties of the molecular complex. To test this requires a detailed and comprehensive molecular network model.

We integrated 57 published synaptic proteomic datasets obtained between 2000 and 2019 that describe over 7000 proteins. The complexity of the postsynaptic proteome is reaching an asymptote with a core set of $\sim 3000$ proteins, with less data on the presynaptic terminal, where each new study reveals new components in its landscape. To complete the network, we added direct protein-protein interaction data and functional metadata including disease association.

The resulting amalgamated molecular interaction network model is embedded into a SQLite database. The database is highly flexible allowing the widest range of queries to derive custom network models based on meta-data including species, disease association, synaptic compartment, brain region, and method of extraction.

This network model enables us to perform in-depth analyses that dissect molecular pathways of multiple diseases revealing shared and unique protein components. We can clearly identify common and unique molecular profiles for co-morbid neurological disorders such as Schizophrenia and Bipolar Disorder and even disease comorbidities which span biological systems such as the intersection of Alzheimer's Disease with Hypertension. 
63

64

65

66

67

68

69

\section{Introduction.}

At neuron-neuron synapses, the proteomes of presynaptic and postsynaptic compartments conduct neurotransmitter release and processing via complex and highly dynamic molecular networks. These signal transduction and plasticity processes underpin normal and abnormal information processing in the brain. The synaptic proteome is physically divided into pre- and postsynaptic subcellular compartments, where the postsynaptic proteome, often referred to as postsynaptic density or PSD, is believed to play a major role in processing and interpretation of the chemical messengers used in synaptic transmission between two neurons.

The PSD has been studied using high throughput proteomic methods for nearly 20 years. Initial smaller datasets (of order tens to hundreds) focused on interactomes associated with specific target proteins (NMDA receptors, AMPA receptors, PSD95, and other MAGUK proteins) [1-6]. Significant increases in the identified protein numbers (order of thousands) were achieved around 2010, when more advanced technologies were applied to whole post synaptic fractions (for example, [7-12]). To address potential noise in the sampling methods, a "consensus" PSD was proposed in 2004 [1] containing 466 proteins that had been reported in multiple studies to date and therefore were proposed as a higher confidence subset.

The pre-synaptic proteome mediates neurotransmitter release through the active zone, which organises the synaptic vesicle cycle through participation in vesicle loading, docking and priming, and calcium-triggering of fusion [13]. The history of the presynaptic proteome spans over 15 years, with the first descriptions in 2004, typically based on synaptic vesicle extraction and purification [14-18].

Proteins usually occur within supramolecular complexes and therefore proteomic datasets are often represented as static undirected protein-protein interaction (PPI) networks. Typically, the network vertices represent biomolecules, such as genes or proteins found in the studies. Edges represent the structural or protein interactions connecting them. Using such a network perspective we can exploit the many statistical measures and fundamental properties from the networks topology to gain insight and make predictions about the underlying data [19]. Vertex centrality 
96 measures such as degree and betweenness reveal influential signalling proteins within

97 the network. The 'scale-free' property [20] found in many biological networks can be

98 used to identify hub genes, which often encode disease related genes [19]. Clustering

99 algorithms attempt to identify densely connected communities (or modules) of

100 vertices within the network [21,22]. Synaptic gene-disease or gene-functional

101 association data can then be annotated onto these communities and tests made for the

102 functional/disease enrichment at the level of the cluster. This can be used to predict

103 gene-sets associated with known synaptic diseases, which are useful elucidating

104 molecular mechanisms[22]. Using this network approach, we can then test where

105 these gene-sets overlap across different disorders.

106

107

108

109 


\section{Results.}

\section{1. Collating the synaptic proteome.}

112

113 We systematically curated synaptic proteomic datasets from the literature, to produce

114 a comprehensive index of the proteins (and their genes) reported at the synapse. To

115 find proteomic studies we searched PubMed using the key words: "synaptic",

116 "postsynaptic', "presynaptic", "synaptosome". Preference was given to the studies

117 focussing on mammalian brains in healthy/normal experimental conditions. The many

118 disease specific studies identified where not included. A total of 57 papers describing

119 a landscape of 7814 synaptic genes (Table 1) were annotated with the following

120 metadata: PUBMED ID, species, method of extraction, number of identified proteins

121 and brain region. Each study's respective protein list was extracted and mapped to

122 stable identifiers (MGI, Entrez and Uniprot) for the predicted set of orthologues for

123 three species (human, mouse, rat). Additional functional information (e.g. GO

124 function, disease association) was overlaid as metadata onto the vertices.

125 Supplementary Table 1 contains a detailed list of the papers found and associated

126 metadata. 


\begin{tabular}{|c|c|c|c|c|}
\hline & PMID & GeneN & Brain region & Specie \\
\hline Husi_2000 & 10862698 & 77 & forebrain & mouse \\
\hline Walikonis_2000 & 108118142 & 29 & forebrain & rat \\
\hline Peng_2004 & 15020595 & 237 & forebrain & rat \\
\hline Satoh_2002 & 11895482 & 45 & forebrain & mouse \\
\hline Youshimura_2004 & 14720225 & 436 & forebrain & rat \\
\hline Farr_2004 & 15447677 & 73 & whole brain & rat \\
\hline Jordan_2004 & 15169875 & 393 & whole brain & mouse and rat \\
\hline Li_2004 & 14532281 & 139 & forebrain & rat \\
\hline Trinidad_2005 & 15748150 & 236 & whole brain & mouse \\
\hline Cheng_2006 & 16507876 & 289 & forebrain and cerebellum & rat \\
\hline Collins_2006 & 16635246 & 620 & forebrain & mouse \\
\hline Dosemeci_2006 & 16332460 & 114 & hippocampus & rat \\
\hline Dosemeci_2007 & 17623647 & 276 & cerebral cortex & rat \\
\hline Trinidad_2008 & 18056256 & 2158 & $\begin{array}{l}\text { cortex, midbrain, cerebellum, } \\
\text { and hippocampus }\end{array}$ & mouse \\
\hline Selimi_2009 & 19402746 & 63 & cerebellum & mouse \\
\hline Fernandez_2009 & 19455133 & 301 & forebrain & mouse \\
\hline Bayes_2001 & 21170055 & 1443 & cortex & human \\
\hline Bayes_2012 & 23071613 & 1552 & cortex & mouse \\
\hline Schwenk_2013 & 22632720 & 34 & whole brain & mouse \\
\hline Distler_2014 & 25211037 & 3558 & Hippocampus & mouse \\
\hline Bayes_2014 & 25429717 & 1134 & Frontal cortex & human \\
\hline Frank_2015 & 2711747 & 145 & forebrain & mouse \\
\hline Uezu_2016 & 27609886 & 928 & Cortex and hippocampus & mouse \\
\hline Focking_2016 & 27898073 & 2021 & Whole brain & human \\
\hline Li_2016 & 27507650 & 1602 & Hippocampus CA1 & mouse \\
\hline Fernandez_2017 & 29045836 & 107 & forebrain & mouse \\
\hline Roy_2017 & 29203896 & 1213 & $\begin{array}{l}\text { frontal, parietal, temporal and } \\
\text { occipital lobes of the } \\
\text { neocortex }\end{array}$ & human \\
\hline Li_2017 & 28671696 & 993 & Hippocampus CA1 & mouse \\
\hline Roy_2018 & 30071621 & 1071 & $\begin{array}{l}\text { frontal, medial and caudal } \\
\text { cortex, right caudate } \\
\text { putamen, right hippocampus, } \\
\text { whole hypothalamus, and } \\
\text { cerebellum (right half) }\end{array}$ & mouse \\
\hline Wilson_2019 & 30986977 & 2134 & cortex & mouse \\
\hline
\end{tabular}




\begin{tabular}{|c|c|c|c|c|}
\hline Coughenour_2004 & 15378707 & 36 & forebrain & rat \\
\hline Blondeau_2004 & 15007177 & 209 & Whole brain & rat \\
\hline Phillips_2005 & 16047384 & 110 & cortex & rat \\
\hline Morciano_2005 & 16269012 & 153 & Whole brain & rat \\
\hline Burre_2006 & 17080482 & 165 & Whole brain & rat \\
\hline Takamori_2006 & 17110340 & 410 & Cerebral cortex & rat \\
\hline Khanna_2007 & 17562281 & 104 & Whole brain & rat \\
\hline Morciano_2009 & 19187093 & 369 & Whole brain & rat \\
\hline Abul-Husn_2009 & 19562802 & 138 & Hippocampus and striatum & mouse \\
\hline Abul-Husn_2011 & 22043286 & 145 & striatum & rat \\
\hline Gorini_2010 & 20114047 & 57 & cortex & mouse \\
\hline Gronborg_2010 & 20053882 & 618 & Cerebral cortex & rat \\
\hline Boyken_2013 & 23622064 & 414 & Cerebral cortex & rat \\
\hline Wilhelm_2014 & 24876496 & 1169 & Cortex and cerebellum & rat \\
\hline Brinkmalm_2014 & 24973420 & 68 & Hippocampus & mouse \\
\hline Weingarten_2014 & 24534009 & 482 & Whole brain & mouse \\
\hline Kokotos_2018 & 30301801 & 983 & cerebellum & rat \\
\hline Filiou_2010 & 20309889 & 2980 & Whole brain & mouse \\
\hline Dahlhaus_2011 & 21398567 & 673 & Visual cortex & mouse \\
\hline Cohen_2013 & 23658807 & 2668 & cortex & rat \\
\hline Biesemann_2014 & 24413018 & 163 & forebrain & mouse \\
\hline Chang_2015 & 25958317 & 2077 & cortex & human \\
\hline Liu_2014 & 25352669 & 1388 & $\begin{array}{l}\text { Hippocampus and prefrontal } \\
\text { cortex }\end{array}$ & mouse \\
\hline Distler_2014 & 25211037 & 4417 & hippocampus & mouse \\
\hline $\begin{array}{l}\text { Kohansal- } \\
\text { Nodehi_2016 }\end{array}$ & 27115346 & 4961 & cerebellum & rat \\
\hline Gonzalez-Lozano & 27748445 & 1560 & cortex & mouse \\
\hline Alfieri_2017 & 28713243 & 351 & telencephalon & mouse \\
\hline Heo_2018 & 29610302 & 2272 & Cortex and hippocampus & mouse \\
\hline
\end{tabular}

144 Table 1: Collection of 57 synaptic proteome studies at a glance. Postsynaptic studies

145 are shown in blue, presynaptic in green and synaptosomal in yellow. 
148 We identified 30 major post synaptic proteome (PSP) studies published in a period spanning 2000-2019 that contribute a total of 5568 mouse and human unique gene

150 identifiers (see Supplementary Table 1). Most of these PSP datasets were obtained by

151 proteomic analysis of biochemical fractions of synaptosomes obtained from

152 homogenised brain samples [3-12, 23-28].

154 Further studies were added that focussed on specific protein complexes using

155 immune, peptide, transgenic affinity purification or in vivo affinity purification

156 approach strategies against well-known PSD proteins, e.g. mGluR5 [29], PSD-95 [2,

157 25, 26, 30], NMDA [1, 2, 31] and AMPA receptors complex [2, 32] or gephyrin [30].

159 The majority of PSP samples were collected from whole brain [3, 10, 29, 32, 33] and

160 forebrain [1, 2, 4-6, 23, 27, 34, 35]. However, several studies focus on specific brain

161 regions, e.g hippocampus [12, 24, 36, 37] and cerebellum [23, 25]. Several studies

162 that considered multiple distinct brain regions simultaneously were also included [11,

$16338,39]$.

164

165 The discovery rate of new PSD proteins was analysed across the multiple studies as

166 shown in Figure 1A, where the number of proteins is plotted against the frequency of

167 identification. Figure 1B shows the ratio of newly identified proteins (black) per year

168 compared to the total number of PSP proteins identified in that year (blue). Two

169 major jumps in the gross number of proteins identified occur in 2008, when 1249 new

170 proteins were reported by [11] and in 2014 with 2588 new proteins added by [12].

171 The most frequently found proteins (i.e. detected in 22, or more, studies out of the 29)

172 include very well-known PSD proteins, for example: DLG4 (found 28/29), CAMK2A

173 (27/29), INA (26/29), SPTBN1, CAMK2B, DLG2, NSF, GRIN2B, GRIN1 (25/29),

174 BIAP2, BSN (24/29) (full list in Supplementary Table 2).

176 High throughput proteomic techniques are powerful, but they are noisy, and

177 contamination is always a concern. A large number (2091) of PSP proteins have been

178 observed just once. While single hits may be accounted for by lack of sensitivity with

179 low abundance molecules, it could also indicate the presence of false positive

180 components brought in by experimental uncertainty. 
182 The rate of growth with respect to newly discovered proteins appears to be slowing

183 (Figure 1B) and therefore there is now an opportunity to define a more reliable subset.

184 Following the approach described in [1], we selected genes found in two or more

185 independent studies to designate the "consensus" PSP. This resulted in 3441 genes,

186 which is $\sim 7$ times larger than reported by [1] and describes a subset of synaptic

187 proteins for which have higher confidence. In this subset we observe the increment of new genes per year decreases after 2008 and drops completely after 2014 (Figure 1C).

189 Seeing that the accumulated number of consensus PSP genes is plateauing, we performed a non-linear fit to extrapolate a predicted total number of consensus PSP genes (Figure 1F, Methods). From the fit, we predict a total number of consensus PSP genes to be 3499 ( $\mathrm{P}=2.36 \mathrm{E}-11$, residual standard error: 192.7 on 12 degrees of freedom) (Figure 1F) by year 2023 which, when compared to the current number

194 (3438) indicates that our knowledge on PSP components, based on currently available methodologies, is close to saturation.

196
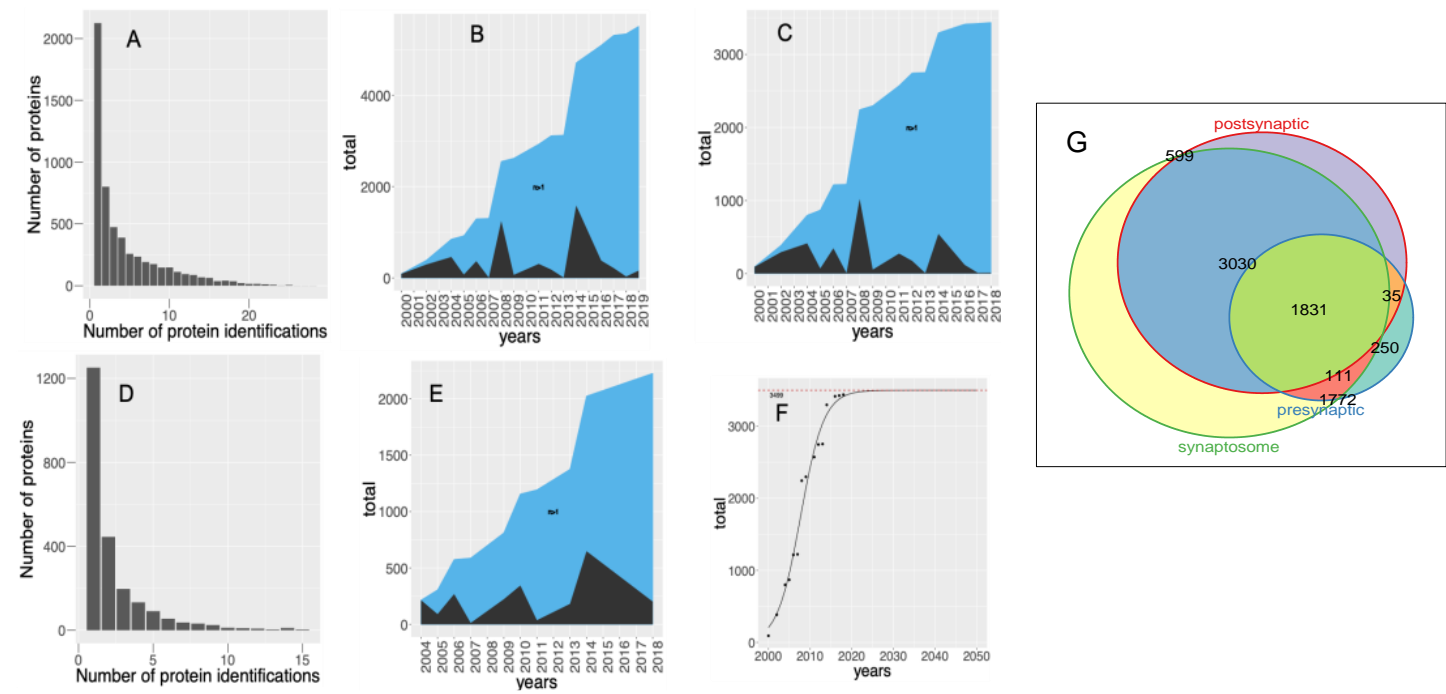

Figure 1. Accumulation of the unique synaptic genes by year. A. Frequency of protein identification in 29 PSP studies. B. Cumulation of the new PSP genes (black) compared to the total datasets (blue) over years. C. Accumulation of new

201 “consensus" PSP genes (black) compared to the total datasets (blue) over years. D.

202 Frequency of protein identification in 17 presynaptic studies. E. Accumulation of new 203 presynaptic genes (black) compared to the total datasets (blue) over years. F. Non204 linear fit to predict the total size of “consensus" PSP (3499 genes) $(P=2.36 E-11)$. 


\subsection{Presynaptic compartment.}

209 For the presynaptic compartment we identified 17 published studies spanning a period

210 from 2004 to 2018, which contribute to 2315 unique human and mouse gene IDs

211 (Supplementary Table 1). As with the PSP studies, presynaptic studies can be

212 methodologically sorted into shotgun and target specific approaches. Shotgun

213 approaches include the majority of studies (14/17), which span differing fractions of

214 presynaptic compartment. This includes studies of the entire presynaptic nerve

215 terminal and plasma membrane $[16,40,41]$ to more specific studies of clathrin coated

216 vesicles (CCV) [42], presynaptic particle fraction (PPF) [43], synaptic vesicles (SVs)

217 [14-18, 44-47], and activity -dependent bulk endocytosis (ADBE) [48]. The three

218 remaining studies consider target protein complexes obtained by Co-IP for N-type

219 calcium channels (CaV2.2/CACNA1B) [49], SNAP -25 [50, 51], BKCa, dynamin-1,

220 SNAP-25, syntaxin-1A, and VAMP-2 [51]. Presynaptic studies span a variety of

221 anatomical regions from whole brain [15-18, 42, 49] to subregion specific, such as

222 cortex [14, 43, 45-47, 51], hippocampus [40, 50] and striatum [40, 41].

224 The frequency of identification of the presynaptic proteins is shown in Figure 1D.

225 From Figure 1E, we see two jumps in newly discovered proteins correspond to studies

226 performed in years 2010 and 2014. Approximately half of the proteins in the

227 presynaptic proteome (1064 genes) have been reported more than twice, which can be

228 viewed as a rough estimate for the consensus of presynapse, however the recent trend

229 in newly identified genes indicates that saturation has not been achieved yet (see

230 Methods). The most frequent presynaptic genes found in the majority of the studies

231 include AP2B1, HSPA8, GNAO1, ACTB (15/17), STX1B, ATP6V0A1, STXBP1, 232 ATP1A3, ATP6V1E1, SYT1, GNB1, TUBA1A, VAMP2, NSF, DNM1 (14/17) with

233 full statistics available in Supplementary Table 3.

\subsection{Synaptosome.}

237 Although most studies targeted pre or post synaptic regions specifically we also 238 considered 11 studies that span the whole synaptosome and reported 6888 unique 
genes. These cover most but not all of the genes/proteins reported in the focussed studies (Figure 1G). In summary, 599 of specifically postsynaptic, 250 of published specifically presynaptic and 35 of their joint overlap were not detected in global synaptosome studies. Supplementary Table 4 contains the whole list of 7814 unique

243 synaptic genes classified according their localisation (presynaptic, post synaptic,

244 synaptosomal) based on the 57 studies considered here.

As before, most of the studies (9/11) could be classified as shotgun proteomic experiments where the entire synaptosome is analysed for its molecular components

$248 \quad[12,52-58]$. Two studies describe the molecular structure of specific protein complexes by Co-IP targeting of kinesin (Kif5C and Kif3A) [59] and P140Cap (SRCIN1) [60]. In terms of brain region origin of the sample, the majority of the

251 studies consider hippocampus [12, 55, 58, 59]; visual cortex [53], motor cortex [56], 252 prefrontal cortex [59] and, cerebral cortex [61].

\section{Reconstructing molecular interaction networks.}

256 To reconstruct protein-protein interaction (PPI) networks for the pre- and post-

257 synaptic proteomes we used human PPI data obtained from three databases: BioGRID 258 [62], Intact [63] and DIP [64]. These data were filtered for the highest confidence 259 direct and physical interactions (see Methods; Table 2), for which we excluded all 260 predicted interactions and those obtained from Co-IP experiments. The resulting PSP 261 network contains 4,817 nodes and 27,788 edges in the Largest Connected Component 262 (LCC). The presynaptic network is significantly smaller and comprises 1780 nodes 263 and 6620 edges in the LCC.

\begin{tabular}{|l|l|l|l|l|}
\hline $\begin{array}{l}\text { Total N of unique } \\
\text { Human PPIs } \\
\text { extracted from three } \\
\text { databases }\end{array}$ & $\begin{array}{l}\text { N of unique Human } \\
\text { PPIs after filtering } \\
\text { for direct/physical } \\
\text { interaction }\end{array}$ & $\begin{array}{l}\text { N of unique PPIs } \\
\text { in PSP network }\end{array}$ & $\begin{array}{l}\text { N of unique PPIs in } \\
\text { PSP “consensus” } \\
\text { network }\end{array}$ & $\begin{array}{l}\text { N of unique PPIs } \\
\text { in Presynaptic } \\
\text { network }\end{array}$ \\
\hline 407,643 & 126,579 & 28,915 & 14,496 & 6,625 \\
\hline
\end{tabular}

266 Table 2. Summary of protein-protein interactions (PPIs) in this study. PPI numbers 


\section{Using the Synaptic Proteome Model.}

271 We captured the original heterogeneity of the datasets by incorporating the necessary

272 metadata so that they can be used in queries that retrieve specific subsets of interest,

273 e.g. only human, only presynaptic, or, say, only genes obtained in Co-IP experiments

274 from hippocampus. To make the datasets readily accessible based on custom selection

275 criteria we embedded them in a SQLlite relational database.

277 In addition to the proteomic, interactomic data and metadata mentioned above, the

278 database also includes the GO function information for three species: mouse, rat and

279 human, disease annotation for human (based on Human Disease Ontology

280 (HDO)[65]) and GeneToModel table, which links certain synaptic proteins to existing 281 computational models of synaptic plasticity and synaptic signal transduction (Sterratt

282 in preparation). The database structure is shown at Figure 2 and described in detail in 283 respective Methods section.

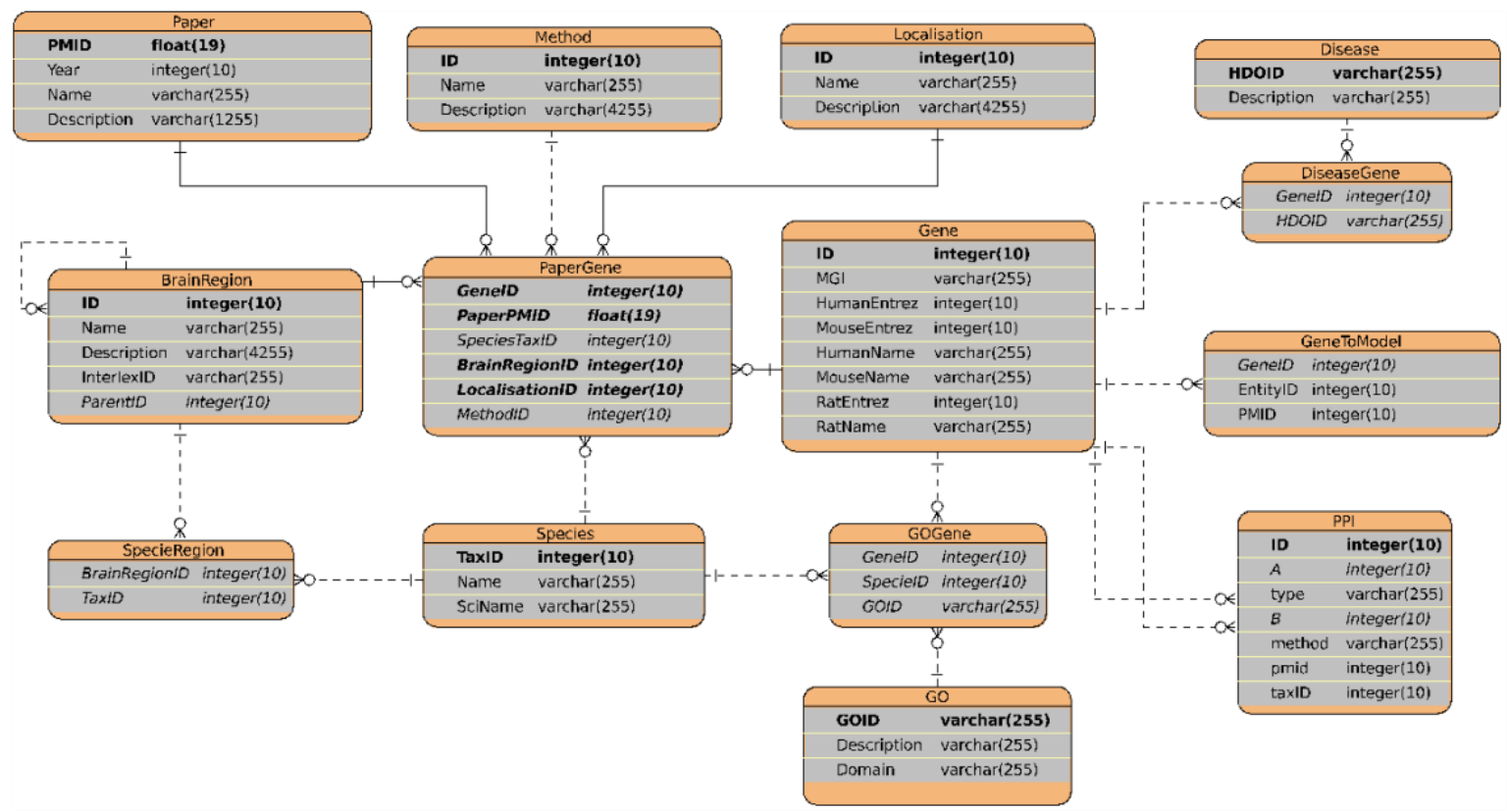

288 Figure 2. Entity Relationship diagram for the SQLite database of the synaptic

289 proteome. 
291 We provide the data as simple flat tables for maximum flexibility and a SQLlite implementation. For anyone who wishes to explore the data we also provide a simple

293 walkthrough of how to install the system and run the following common uses/queries

294 from SQLite Studio or R (Supplementary Files). Following use cases illustrate how

295 the database can be used to obtain the information for specific protein(s) and build the

296 PPI network for subset of proteins $(1,2)$, and how the PPI network can be used for

297 further analysis $(3,4)$.

298 Database is available from Supplementary Data.

\subsection{Finding information for specific gene/s of interest.}

302 Among the common questions in the neuroscientific community are "What is known

303 about my favourite gene/protein? Is it pre- or postsynaptic? Which brain region it was

304 found and by whom?". Evidence to address these types of questions can be extracted by simple database queries using protein name (gene name) or gene Entrez ID.

306 For example, to find the information for protein "SRCIN1", the query will look like:

HumanName,

YEAR,

320 Here, FullGeneFullPaperFullRegion is a database view that combines the major

321 information for all genes in more convenient spreadsheet style representation. 
323 The database returns the results in a form of table (Table 3 contains top 17 rows),

324 showing that SRCIN1 was found in 25 postsynaptic, 7 presynaptic and 12

325 synaptosomal studies, along with brain regions where it was reported.

327 Table 3 A snippet of available information for SRCIN1

\begin{tabular}{|c|c|c|c|c|c|}
\hline Compartment & EntrezID & GeneName & PMID & AUTHOR_YEAR & Brain region \\
\hline Postsynaptic & 80725 & SRCIN1 & 15447677 & FARR_2004 & Brain \\
\hline Postsynaptic & 80725 & SRCIN1 & 15020595 & PENG_2002 & Forebrain \\
\hline Postsynaptic & 80725 & SRCIN1 & 18056256 & TRINIDAD_2008 & Midbrain \\
\hline Postsynaptic & 80725 & SRCIN1 & 18056256 & TRINIDAD_2008 & Cerebellum \\
\hline Postsynaptic & 80725 & SRCIN1 & 30071621 & ROY_2018 & Hypothalamus \\
\hline Postsynaptic & 80725 & SRCIN1 & 16332460 & DOSEMESI_2006 & Hippocampus \\
\hline Postsynaptic & 80725 & SRCIN1 & 30071621 & ROY_2018 & Striatum \\
\hline Postsynaptic & 80725 & SRCIN1 & 17623647 & DOSEMESI_2007 & Cerebral cortex \\
\hline Postsynaptic & 80725 & SRCIN1 & 29203896 & ROY_2017 & Frontal lobe \\
\hline Presynaptic & 80725 & SRCIN1 & 24876496 & WILHELM_2014 & Cerebellum \\
\hline Presynaptic & 80725 & SRCIN1 & 17110340 & TAKAMORI_2006 & Cerebral cortex \\
\hline Synaptosome & 80725 & SRCIN1 & 27115346 & KOHANSAL_NODEHI_2016 & Cerebellum \\
\hline Synaptosome & 80725 & SRCIN1 & 28713243 & ALFIERI_2017 & Telencephalon \\
\hline Synaptosome & 80725 & SRCIN1 & 25211037 & DISTLER_2014 & Hippocampus \\
\hline Synaptosome & 80725 & SRCIN1 & 27115346 & KOHANSAL_NODEHI_2016 & Cerebral cortex \\
\hline Synaptosome & 80725 & SRCIN1 & 25352669 & LIU_2014 & Prefrontal cortex \\
\hline Synaptosome & 80725 & SRCIN1 & 25958317 & CHANG_2015 & Motor cortex \\
\hline Synaptosome & 80725 & SRCIN1 & 21398567 & DAHIHAUS_2011 & Visual cortex \\
\hline
\end{tabular}

\subsection{Building a custom PPI network from a protein list.}

332 We can easily extract a customised subset of the PPI network that includes specific

333 studies, brain regions or compartments. The example query for a presynaptic protein

334 list and its respective PPI network is as follows:

SELECT DISTINCT A,

\section{FROM PPI}

339 JoIN FullGeneFullPaper fl oN fl. GeneId= A

340 JoIN FullGeneFullPaper f2 on f2. GeneId =B 
WHERE f1. Localisation= 'Presynaptic'

AND f2. Localisation ='Presynaptic'

344 This will result in a list of protein interaction pairs of type "Human_Entrez_ID_A - -

345 Human_EntrezID_B”, which can be visualised in R, using package Igraph

346 (https://igraph.org/redirect.html), loaded to Cytoscape via RCy3 package [66] or

347 saved as csv file and analysed with Cytoscape (https://cytoscape.org/) or Gephi

348 (https://gephi.org/).

3.3. Measuring how network topology correlates with functional/disease importance: Bridgeness.

The relative importance of specific proteins to propagate information (i.e. signals) through a network can be estimated from the position of the protein within the network's architecture and its substructures. The number of proteins interacting neighbours (degree) is considered as important property as it may reflect protein

358 importance for signal propagation in the network. This can be extended to include

359 local interacting partners, a measure known as Semi-local centrality [67].

360 Clustering is commonly used to identify substructures/communities within networks.

361 Concrete clustering methods usually assign each protein to the single most likely

362 community despite many of them actually being involved in multiple communities.

363 To match this we calculate the probability of each protein being involved in every

364 cluster in the network. The more communities a specific protein "bridges" (has a

365 probability belonging to) the more likely it is involved in communicating between

366 these communities across the network [68] (Methods). We hypothesise these

367 'Bridging' proteins will also correlate with functional/disease importance at the

368 biological level. 


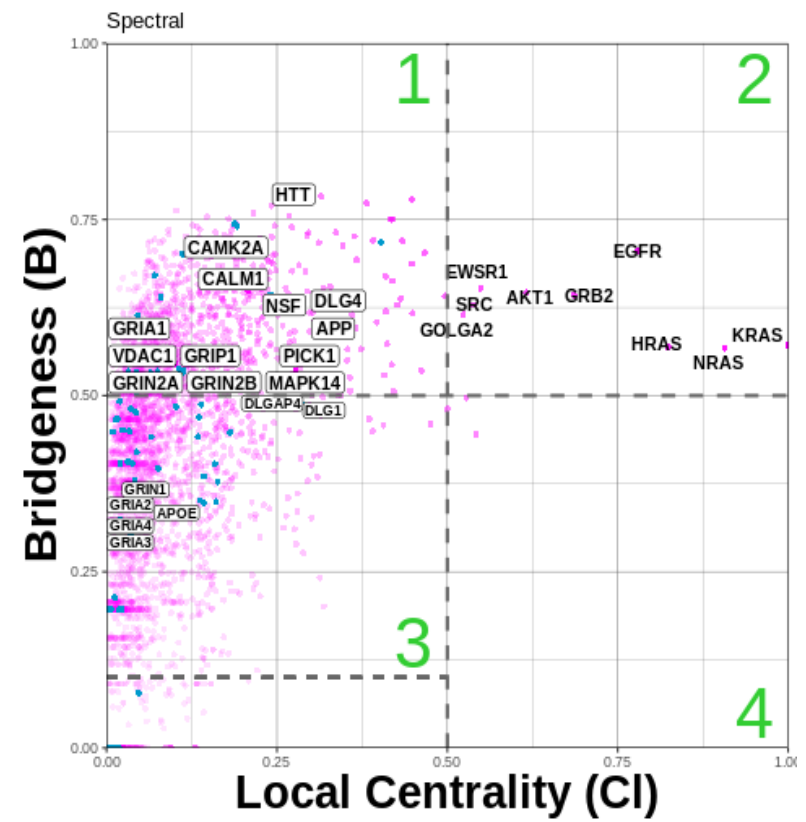

388

Figure 3. Bridging proteins estimated using the Spectral clustering algorithm. Bridgeness is plotted against semi-local centrality (Methods), allowing categorisation of the proteins to three important groups: Region 1, proteins that are likely to have a 'global' rather than 'local' influence in the network (also been called bottle-neck bridges [69], connector or kinless hubs [70] lie in the range (DLG4, GRIN2B, $C A M K 2 A$, etc). Region 2, proteins likely to have an influential 'globally' and 'locally' in the network (EGFR, HRAS, NRAS, etc). Region 3, more likely to be centred within the community they're found in, but also focused on communicating with a few other specific communities (GRIN1, GRIA2-4), "secondary bridging proteins". Region 4, proteins whose impact is mostly 'locally', primarily within one or two communities (local hubs [68]).

Using the Spectral clustering algorithm [71], we estimated the Bridgeness for the PSP network and identified a total of 3884 (3884/4817 80\%) proteins with Bridgeness value more than 0.1 .

We then plotted Bridgeness against Semi-local centrality values (see Methods) to categorise the proteins by their ability to influence the network globally and locally (Figure 3, Supplementary Table 5). Region 1 contains 1126 proteins (see Region 1 in Figure 3, Supplementary table 5), which, between them affect many (i.e. greater than half) communities in the network yet each is relatively localised in the network (low local-centrality values) - we call these "bridging" or "influential". Of those 1126 - 28 are considered as core PSD95/DLG4 interactors [34] (28/77 36\%) within the postsynaptic proteome including: DLG4, GRIN2A, GRIN2B, CAMK2A, CALM1, NSF. Interestingly, we found NMDA-receptor subunits proteins GRIN1 and AMPAreceptor subunit proteins (GRIA2,3,4) clustered together as secondary Bridging proteins (see Region 3 in Figure 3), indicating their influence on a small number of selected functional clusters. There are a few scaffold proteins which bind to the AMPA-receptor subunits which are Bridging Proteins, e.g. DLG1, PICK1, GRIP1. 
409

410

411

412

413

414

415

416

417

418

419

420

421

422

423

424

425

426

427

428

429

430

431

432

433

434

435

436

437

438

439

440

Among 1126 Bridging proteins, $512(512 / 1126 \sim 45 \%)$ were found associated with at least one synaptopathies given in our set (Methods), $248(214 / 1126 \sim 19 \%)$ with multiple diseases including: APP (AD\&Epi\&ASD\&PD\&HTN\&MS\&FTD), VDAC1 (AD\&PD\&MS), and MAPK14 (AD\&SCH\&HD\&HTN\&MS); Indeed, using the Spectral algorithm we found significant overlaps between the Bridging proteins (see Region 1 in Figure 3) and disease association; AD (P=7.74E-10,***), HTN (P=1.16E07***), HD ( $\mathrm{P}=8.01 \mathrm{E}-7 * * *), \mathrm{PD}(\mathrm{P}=7.9 \mathrm{E}-5 * * *)$. Moreover, when we considered synaptic function (GO annotation), we found a significant overlap for $\mathrm{AD}$ with innate immune response-activating signal transduction (GO:0002758, $\mathrm{AD}=4.3 \times 10-11$, $\mathrm{HTN}=9.7 \times 10-10)$, stress-activated MAPK cascade (GO:0051403, AD=3.310-12, $\mathrm{HTN}=4.3 \times 10-11)$ and calcium-mediated signaling (GO:0019722, $\mathrm{AD}=1.1 \times 10-3)$.

\subsection{Extracting and comparing shared disease pathways.}

Given gene-disease annotation (GDA) data and the PPI network topology, we can start to dissect how these different neurological diseases might coalesce at the synapse. The methodology described in [72] tests the extent to which two diseases might share a common molecular mechanism. Briefly, within the network we calculate z-scores from the observed mean shortest distance between disease GDA's, relative to 10000 random permutation of each diseases GDA's, to quantify the disease pairs overlap (Methods). From the set of p-values, calculated from these z-scores, we then correct for false positives by calculating the q-values (Supplementary Table 6).

Figure 4 shows the comparison of the disease pair overlaps obtained for common neurological and neurodevelopmental diseases and disorders in pre- and postsynaptic networks. It can be seen that the overlap for common neuropsychiatric/developmental disorders was observed in both postsynaptic and presynaptic PPI network models (post/pre), however q-values are smaller for presynaptic network, probably due to its size: BP-ASD (P=8.79E-14/1.44E-03), BP$\mathrm{SCH}(\mathrm{P}=2.65 \mathrm{E}-25 / 9.55 \mathrm{E}-11)$, ASD/SCH (P=8.74E-07/9.82E-03). Similarly, overlap was observed for common neurodegenerative diseases/conditions AD and PD $(\mathrm{P}=1.74 \mathrm{E}-5 / 1.92 \mathrm{E}-05)$. 
A

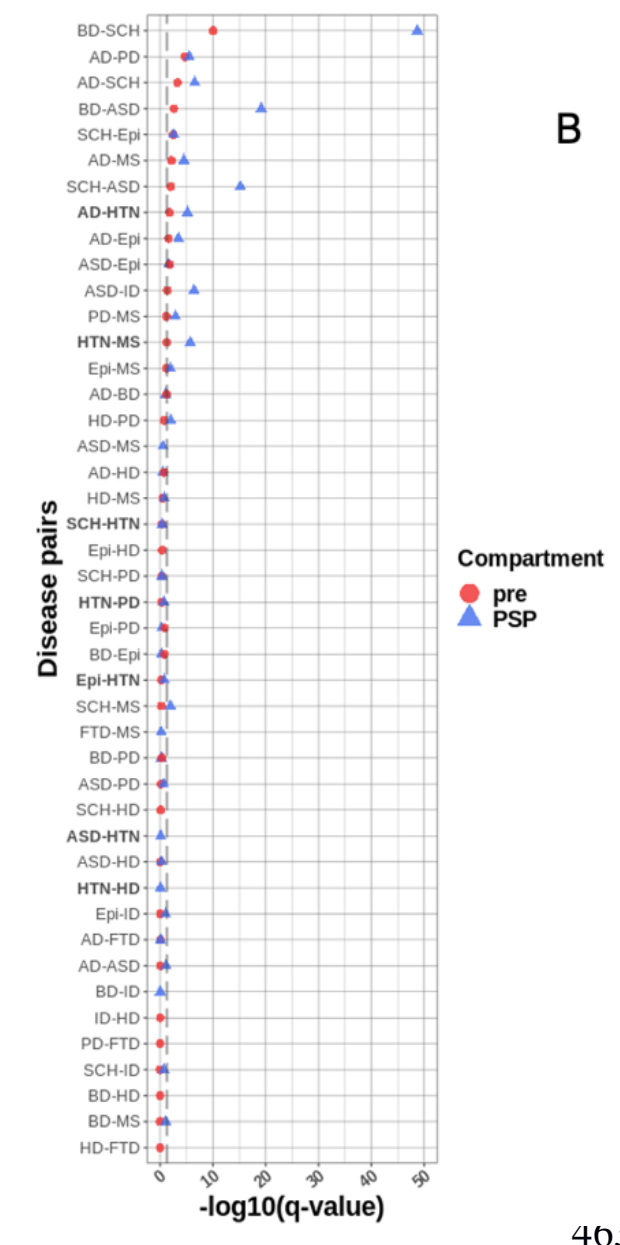

Figure 4. Disease-disease relationship for presynaptic and postsynaptic interactome. Where significance $q$ values $<0.05$ is delineated by the dashed line. Schizophrenia (SCH), Autistic Spectrum Disorder (ASD), Autistic Disorder (AUT), Bipolar Disorder (BD), Intellectual Disability (ID), Alzheimer disease (AD), Epilepsy Syndrome (Epi), Parkinson's Disease $(P D)$, Frontotemporal Dementia (FTD), Huntington's Disease (HD) and Multiple Sclerosis (MS) are considered.

465 Hypertension (HTN) is considered one of the leading vascular risk factors associated 466 with neurological diseases/disorders including AD, PD and FTD [73-75], and BD and 467 SCH [76, 77]. With the PSP PPI network model, we found a strong overlap for HTN 468 with $\mathrm{AD}(\mathrm{P}=8.6 \mathrm{E}-4$, and with $\mathrm{MS}(\mathrm{P}=8.79 \mathrm{E}-5)$, but not with the other neurodegenerative diseases: FTD-HTN, PD-HTN, Epi-HTN and HD-HTN which all have q-values $>0.05$ (Supplementary Table 6). When considering the set of common neuro-psychiatric/ developmental disorders with HTN, we found all four overlap with HTN, i.e., but that there was no evidence these overlaps were significant when considering a full randomised model: HTN-SCH ( $\mathrm{P}=0.9), \mathrm{HTN}-\mathrm{BD}(\mathrm{P}=0.9), \mathrm{HTN}-\mathrm{ID}$ $(\mathrm{P}=0.32)$ and HTN-ASD $(\mathrm{P}=1)$.

476 The more a disease pair overlaps, the greater the evidence the corresponding disease

477 pair share the same genes, and therefore participate in similar pathways. Since we find 478 significant overlaps between AD-HTN and AD-PD, but not PD-HTN, it indicates a 
potential shared mechanistic pathway between AD and HTN, which is different to the pathways shared between AD and PD; Supplementary Figure 3A and 3C illustrates this difference by plotting the z-score calculated against the distribution of for ADHTN and PD-HTN after 10000 randomised models using the PSP network.

\section{Discussion.}

By systematic interrogation of the published literature we identified 57 studies that describe a molecular landscape of the synaptic proteome comprising 7617 unique mouse and human genes. Of this, the majority of the papers (29/57) are for the postsynaptic compartment, 17 are presynaptic and 11 consider the whole synaptosome. We combined all the datasets based on stable identifiers, which resulted in 5568 postsynaptic genes (3441 found in two or more studies forming an updated “consensus" PSP), 2315 presynaptic genes, and 6888 reported for synaptosome. We retrieved the PPIs for the list of genes, which allows us to build a comprehensive PPI map for the whole synapse or its subcellular compartments.

We embedded the datasets in a SQLite database to enable more flexible queries based on the species, brain region, method of identification or other metadata and allow the extraction of bespoke protein network models for subsequent analyses.

This dataset is the largest and the most complete up to date and is freely available with lightweight tools to allow anyone to extract relevant subsets. By mirroring the methods used it would be straightforward for any user to add in their own datasets for comparison.

The case studies described provide useful examples of the analyses that can be readily extracted from these data. In addition, to information for specific proteins, we can use the model to analyse the entire protein-protein interaction network to query topologyfunction relationships that provides insights for possible disease mechanism. For example, since the Bridging proteins are spread out over the network, the correlated disease-disease pairs we find are not centred in a single cluster but shared across several communities. In turn, the co-occurrence of enrichment of specific synaptic functions with disease in the discovered communities may indicate that molecular 
511 complexes underlying the specific synaptic functions are also involved in disease

512 mechanisms. When comparing across diseases we find evidence for shared molecular

513 mechanisms that span common neurological disorders, such as Bipolar disease and

514 Autism Spectrum Disorder, Bipolar Disease and Schizophrenia. Moreover we see

515 evidence for molecular mechanisms that span more diverse disease pairs such as the

516 existence of common molecular pathways linked to both Alzheimer's disease and

517 Hypertension.

518 Methods.

519 1. Data cleaning and ID mapping

520 Synaptic studies for presynaptic, postsynaptic and whole synaptosome compartment

521 were identified by manual curation of PubMed, starting from year 2000. Protein/gene

522 lists from the published synaptic proteome studies were combined into the total list of

523 synaptic components. Identifiers from each study were mapped to stable IDs

524 including: Entrez Human and Mouse, Uniprot and MGI IDs. The resulting 'master list' 525 contains only unique gene entries (rows) with corresponding studies (columns) where

526 they have been identified. For example, if a protein was discovered in several studies

527 all those respective columns will contain 1. A summary of all studies is presented in

528 Supplementary Table 1. It contains the information for literature ID and some useful

529 metadata such as species (mouse, human, rat), protein counts, brain region where the

530 sample was taken from along with experimental methods used for protein

531 identification and quantification.

2. Prediction of total size of pre- and postsynaptic proteome.

534 Only proteins that were found more than one time were taken into account to make

535 the most confident "consensus" dataset for pre-and post-synaptic proteomes.

536 We fitted the accumulation of new proteins against the year they were first time

537 identified in $\mathrm{R}$, using linear ( $\sim \mathrm{x})$, and non-linear (y $\sim$ SSlogis ( $\mathrm{x}$, Asym, $\mathrm{xmid}, \mathrm{scal})$ )

538 models. The goodness of fit was compared by Akaike's Information Criteria) AIC

539 function [78], where lower indicates a more parsimonious model.

541 For post synaptic proteome the non-linear model is shown at Figure 1F and predicted 542 maximum size of “consensus" PSP proteome is 3499, achieved by roughly 2023. 
543 AIC coefficient is 213.8955 for linear fit and 205.0504 for non-linear fit, which means

544 the latter is more parsimonious.

546 For presynaptic proteome the non-linear fit is shown at Supplementary Figure 1,

547 predicting 1309 proteins in total reached by 2035 year.

548 However, by AIC criteria, the liner model for presynaptic "consensus" proteome is

549 better than non-linear (103.2001 and 107.2766, respectively), which likely means that

550 presynaptic proteome is not in its "saturation" phase yet.

\section{3. Protein-protein interaction data}

553 Protein-Protein Interaction (PPI) dataset was assembled from three publicly available 554 databases: DIP [62]. IntAct [61] and BioGRID [60]. The interactions were retrieved in 555 PSI-MITAB standard protein interaction format $[74,75]$. The PSI-MITAB format 556 enforces unified column contents and use of controlled vocabularies. These columns 557 report different types of identifiers of an interactor pair, the PubMed identifier of 558 publication where the interaction was reported, taxonomies of interactors, an 559 interaction detection method, and an interaction type. The last two identifiers are 560 defined with unique MI-terms specified in the Molecular Interactions Controlled 561 Vocabulary (MI) ontology. The set of MI-terms representing each of the categories 562 was obtained by parsing the MI ontology file (source:

563 https://www.ebi.ac.uk/ols/ontologies/mi).

565 To limit the analysis to high-confidence direct physical interactions, those annotated 566 by "association" term (MI:0914) and its offspring were preserved. This step excluded 567 interactions of types: colocalization, functional association, genetic interaction and 568 predicted interaction. Although MI ontology offers "direct interaction" term in the 569 category of interaction type, according the the iMEX curation rules

570 (https://github.com/IMEx-Consortium/IMEx-site/raw/gh-

571 pages/static/files/imex_curation_rules.pdf) two hybrid assays are categorised as non572 direct interactions. Therefore, this classification was not used to preserve interactions 573 reported by two-hybrid assays. We further removed indirect interactions, often linked 574 to methods likely to generate false positive hits (spoke-expansion). Interactions 575 originating from experiments involving co-complexes (e.g. pull-down, affinity 
technology) were excluded from the analysis by filtering out a selected subset of terms denoting these methods. Since the intAct interaction table indicates spokeexpanded interactions, these were also removed from the interaction set.

Integration of interaction tables required distinct parsing procedures per database, including translation into common interactor identifiers. DIP and IntAct databases use UniProtKB accessions (UniProtKB ACs) as their primary identifiers, e.g. P49418. On the other hand, BioGRID uses numeric Entrez Gene IDs, e.g. 1134. The unification of identifiers was achieved by combining mapping tables collected from NCBI and UniProtKB ftp servers ( file addresses:

ftp://ftp.uniprot.org/pub/databases/uniprot/current_release/knowledgebase/idmapping/ by_organism/RAT_10116_idmapping_selected.tab.gz ftp://ftp.uniprot.org/pub/databases/uniprot/current_release/knowledgebase/idmapping/ by_organism/HUMAN_9606_idmapping_selected.tab.gz ftp://ftp.uniprot.org/pub/databases/uniprot/current_release/knowledgebase/idmapping/ by_organism/MOUSE_10090_idmapping_selected.tab.gz ftp://ftp.ncbi.nlm.nih.gov/gene/DATA/gene2accession.gz). Entrez Gene ID was chosen as a unifying identifier.

Interactors that were annotated with a different taxon than Mouse, Rat or Human were removed. Non-human Entrez Gene IDs were mapped to Human identifiers with gene orthology mapping tables available on NCBI ftp server (file address: https://ftp.ncbi.nlm.nih.gov/gene/DATA/gene_orthologs.gz). After application of the above criteria, 407443 interactions were obtained. Additionally, after manual curation, 200 interactions from PDB database were added directly to the PPI list with Method "MI:0114" - X-ray crystallography, and Type “MI:0407”- Direct interaction, which resulted in total of 407643 interactions.

All files where retrieved on the same date, 2020-06-18, parsed and integrated with a custom code available at https://gitlab.com/Wysocka/ppis_v02.

\section{Estimation of Bridgeness and semi- local centrality measures.}


609 To assess the topological importance of the proteins in PSP network we estimated two

610 independent measures for each protein.

611 1) semi-local centrality $\mathrm{Cl}(\mathrm{v})$, which takes into consideration both a vertex's degree,

612 its nearest, and next to nearest neighbours:

$$
\begin{aligned}
& Q(u)=\sum_{w \in \Gamma(u)} N(w) \\
& C l(v)=\sum_{u \in \Gamma(u)} Q(u)
\end{aligned}
$$

where $\Gamma(u)$ is the set of nearest neighbours of $u$ and $N(w)$ is the number of nearest and next to nearest neighbours of the vertex $w$. We performed unity-based or feature

621 range $[0,1]$.

622 Semi-local centrality differs from degree centrality therefore, in making use of more

623 information, allowing us to also measure a vertices' spread' of information locally

624 through the network.

625 2) Bridgeness $B(v)$ of vertex $v$ to measure the influence of a gene due to the clustering $626[68]$ can be estimated as:

$$
B(v)=1-\sqrt{\frac{c}{c-1}\left(\sum_{j=1}^{c} u_{j v}-\frac{1}{c}\right)^{2}}
$$

630 Where $u_{v}$ is the community membership vector for vertex $v$, which is the probability

631 of vertex $v$ belonging to a given community $u_{v}=\left[u_{1 v}, u_{2 v}, \ldots, u_{c v}\right]$,

632 where $\sum_{j} u_{j v}=1$ and $\mathrm{c}$ is the number of communities detected by the algorithm.

633 The combination of these measures allows categorisation of the influence each has on

634 the overall network structure (Figure 3):

\section{Disease Network Localisation}

We compared the gene-disease association for the pre and post-synaptic PPI networks using the combined OMIM/GeneRIF/Ensembl variation data for common set of synaptic diseases (or synaptopathies) [79]: Schizophrenia (SCH, DOID:5419), Autistic Spectrum Disorder (ASD, DOID:0060041), Autistic Disorder (AUT, 
DOID:12849), Bipolar Disorder (BD, DOID:3312), Intellectual Disability (ID, DOID:1059), Alzheimer disease (AD, DOID:10652), Epilepsy Syndrome (Epi, DOID:1826), Parkingson's Disease (PD, DOID:14330), Frontotemporal Dementia (FTD, DOID:9255), Huntington's Disease (HD, DOID:12858), Multiple Sclerosis (MS, DOID:2377) and Hypertension (HTN, DOID:10763).

We investigated the overlap and separation of each disease-disease pair by measuring the mean shortest distance for each disease, using the shortest distance between each GDA to its next nearest GDA neighbour [72]. The overlap, or separation, of each disease-disease pair in the pre- post-synaptic PPI networks, could then be quantified using:

$$
S_{A B} \equiv\left\langle d_{A B}\right\rangle-\frac{\left\langle d_{A A}\right\rangle+\left\langle d_{B B}\right\rangle}{2}
$$

Where $\left\langle d_{A A}\right\rangle$ and $\left\langle d_{B B}\right\rangle$ quantify the mean shortest network distance between genes associated with disease A (or B), and $\left\langle d_{A B}\right\rangle$ the mean shortest distance between diseases. $S_{A B}$ is bound by the diameter of the network, i.e., $d_{\max } \leq S_{A B} \leq d_{\max }$ where $d_{\text {max }}$ is $8,7,8$ for the presynaptic, PSP and PSP consensus PPI networks respectively. The magnitude of $S_{A B}$ depends on the number of GDSs associated with each disease. Large positive values imply two well separated diseases, while large negative values indicate large (number of GDAs) diseases with a big overlap, often implying one disease is the variant or precursor to the other. Each disease-disease network separation pair $\left(S_{A B}\right)$ was compared against a full randomised model: drawing the same number of GDAs (from the set of all network genes) for each disease at random, before computing its separation. For each disease-disease pair, we performed 10,000 iterations of the full randomised model using the ECDF distributed computing facility.

The difference between the observed and randomised disease pair separations, was quantified using the z-score:

$$
z-\operatorname{score}_{A B}=\frac{S_{A B}-\left\langle S_{A B}^{\text {rand }}\right\rangle}{\sigma\left(S_{A B}^{r a n d}\right)}
$$

671 Where $\left\langle S_{A B}^{\text {rand }}\right\rangle$ and $\left(\sigma_{A B}^{r a n d}\right)$ are the mean and standard deviation obtained from the 67210,000 iterations. Each disease-disease pair separation using the full randomised 
673 model, i.e., $S_{A B}^{r a n d}$, was found to follow a normal distribution. We therefore assessed

674 the significance of each disease-disease pair's separation, from P-values estimated

675 from its Z-score calculated in (4):

676

$$
P-\operatorname{value}\left(X=-\left|Z-\operatorname{score}_{A B}\right| ; \mu=0, \sigma=1\right)=\left[1+\operatorname{erf}\left(\frac{x-\mu}{\sigma \sqrt{2}}\right)\right]^{-}
$$

679 Where we take the negative of the absolute value of each disease-disease pairs z-score

680 calculated in (4) and make use of R's pnorm function available in the 'stats' package

681 ( $\mathrm{R}$ version 3.4.2).

682 The confidence in each disease-disease pairs P-value was tested for by calculating its 683 q-value [80], and from the Bonferroni correction at the $0.05(*), 0.01(* *)$ and 0.001 $684 \quad(* * *)$ significant levels.

\section{SQL database}

The database contains the following main tables:

689 - Gene: list of genes including IDs (MGI, Entrez Human and Mouse) and gene

690 names (Human and Mouse).

$691 \quad$ - Specie: Tax ID (Human and Mouse)

692 - Paper: list of papers with PMID ID, name (in format "FirstAuthor_year"), year 693 of publication

694 - Location: postsynaptic, presynaptic, synaptosome

695 - Method: shotgun or IP

696 - Brain region: list of regions where the samples originate, with hierarchical

697 region structure

698 - PPI: human protein-protein interactions combined from BioGRID, Intact and

699 DIP databases, contain information on methods, interaction type (PSI-MI

700 nomenclature) and PMID info for each of the interactions.

701 - PaperGene: table links gene to respective papers and the metadata above

702 - GO: BP, CC and MF GO annotation for Human, Mouse and Rat species

703 - GOGene: gene to GO association list

704 - Disease: List of diseases from the HDO for Human

705 - DiseaseGene: genes to disease association list 
706 - GeneToModel: genes with found association with published model of synaptic

707 plasticity

708 The database is created with SQLite v 3.31.1 RDBMS in SQLite Studio v3.2.1.

709

710 Acknowledgements:

711 OS, CM, JDA, DS, were funded by European Union Horizon 2020 Specific Grant

712 Agreement No. 72027 (Human Brain Project SGA2). OS and JDA were funded by

713 European Union Horizon 2020 Specific Grant Agreement No. 945539 (Human Brain

714 Project SGA3). The work of S.G.N.G. was supported by the UK Medical Research

715 Council (G0802238, Defining the Human Synapse Proteome) and Simons Initiative

716 for the Developing Brain. The Simons Initiative for the Developing Brain (SIDB) at

717 the University of Edinburgh is supported by the Simons Foundation Autism Research

718 Initiative (529085)

720 Author contribution:

OS, CM, MDC selected and curated the datasets; EW, KFH, XH, TIS designed and performed the PPI extraction and filtering; $\mathrm{CM}$ and OS performed the network analysis, wrote the analysis scripts and performed the statistical testing; OS constructed the SQLite database; OS, CM, JDA, DS, TIS, SGNG designed and directed the study, wrote and edited the manuscript. 


\section{Supplementary Figures and Tables.}

729 Figure 1. Non-linear model for accumulation of presynaptic genes over years (year of

730 first identification is considered), predicted number of 1309 genes, which should be

731 reached by 2035 .

733 Figure 2. The hierarchical structure of brain regions (human) existing in the database, 734 according to 57 selected studies.

736 Figure 3. Linear mapping of Z-scores with ecd function used for assigning of gene 737 compartment localisation.

739 Supplementary Table 1. List of 57 synaptic publications combined for the database 740 with information on year, PUBMID, gene count, method, brain region and short 741 description.

743 Supplementary Table 2. List of 5568 post synaptic genes collected from 29

744 publications listed as columns. 1 or 0 in each row corresponds to "found" or "not 745 found" for each specific gene in each specific publication.

747 Supplementary Table 3. List of 2315 presynaptic genes collected from 17 publications 748 listed as columns. 1 or 0 in each row corresponds to "found" or "not found" for each 749 specific gene in each specific publication.

751 Supplementary Table 4. Full list of 7814 unique synaptic genes classified according 752 their localisation (presynaptic, post synaptic, synaptosomal) based on 57 studies 753 considered here. Localisation gets " 1 " if at least one publication has reported this gene 754 for this compartment.

756 Supplementary Table 5. Estimation of Bridgeness values for PSP network protein.

758 Supplementary Table 6. Estimation of disease pairs overlap. 
References.

762

763

764

765

766

767

768

769

770

771

772

773

774

775

776

777

778

779

780

781

782

783

784

785

786

787

788

789

790

791

792

793

794

795

796

797

798

799

800

801

802

803

804

805

806

807

808

809

1. Collins, M.O., et al., Molecular characterization and comparison of the components and multiprotein complexes in the postsynaptic proteome. J Neurochem, 2006. 97 Suppl 1: p. 16-23.

2. Husi, H., et al., Proteomic analysis of NMDA receptor-adhesion protein signaling complexes. Nat Neurosci, 2000. 3(7): p. 661-9.

3. Jordan, B.A., et al., Identification and verification of novel rodent postsynaptic density proteins. Mol Cell Proteomics, 2004. 3(9): p. 857-71.

4. Li, K.W., et al., Proteomics analysis of rat brain postsynaptic density. Implications of the diverse protein functional groups for the integration of synaptic physiology. J Biol Chem, 2004. 279(2): p. 987-1002.

5. Peng, J., et al., Semiquantitative proteomic analysis of rat forebrain postsynaptic density fractions by mass spectrometry. J Biol Chem, 2004. 279(20): p. 21003-11.

6. Yoshimura, Y., et al., Molecular constituents of the postsynaptic density fraction revealed by proteomic analysis using multidimensional liquid chromatography-tandem mass spectrometry. J Neurochem, 2004. 88(3): p. 759-68.

7. $\quad$ Bayes, A., et al., Comparative study of human and mouse postsynaptic proteomes finds high compositional conservation and abundance differences for key synaptic proteins. PLoS One, 2012. 7(10): p. e46683.

8. Bayes, A., et al., Characterization of the proteome, diseases and evolution of the human postsynaptic density. Nat Neurosci, 2011. 14(1): p. 19-21.

9. Bayes, A., et al., Human post-mortem synapse proteome integrity screening for proteomic studies of postsynaptic complexes. Mol Brain, 2014. 7: p. 88.

10. Trinidad, J.C., et al., Phosphorylation state of postsynaptic density proteins. J Neurochem, 2005. 92(6): p. 1306-16.

11. Trinidad, J.C., et al., Quantitative analysis of synaptic phosphorylation and protein expression. Mol Cell Proteomics, 2008. 7(4): p. 684-96.

12. Distler, U., et al., In-depth protein profiling of the postsynaptic density from mouse hippocampus using data-independent acquisition proteomics. Proteomics, 2014. 14(21-22): p. 2607-13.

13. Chua, J.J., et al., The architecture of an excitatory synapse. J Cell Sci, 2010. 123(Pt 6): p. 819-23.

14. Boyken, J., et al., Molecular profiling of synaptic vesicle docking sites reveals novel proteins but few differences between glutamatergic and GABAergic synapses. Neuron, 2013. 78(2): p. 285-97.

15. Burre, J., et al., Analysis of the synaptic vesicle proteome using three gelbased protein separation techniques. Proteomics, 2006. 6(23): p. 6250-62.

16. Morciano, M., et al., Immunoisolation of two synaptic vesicle pools from synaptosomes: a proteomics analysis. J Neurochem, 2005. 95(6): p. 1732-45.

17. Morciano, M., et al., The proteome of the presynaptic active zone: from docked synaptic vesicles to adhesion molecules and maxi-channels. J Neurochem, 2009. 108(3): p. 662-75.

18. Weingarten, J., et al., The proteome of the presynaptic active zone from mouse brain. Mol Cell Neurosci, 2014. 59: p. 106-18.

19. Vidal, M., M.E. Cusick, and A.L. Barabasi, Interactome networks and human disease. Cell, 2011. 144(6): p. 986-98.

20. Barabasi, A.L. and R. Albert, Emergence of scaling in random networks. Science, 1999. 286(5439): p. 509-12. 
21. Newman, M.E., Modularity and community structure in networks. Proc Natl Acad Sci U S A, 2006. 103(23): p. 8577-82.

\section{Pocklington, A.J., J.D. Armstrong, and S.G. Grant, Organization of brain} complexity--synapse proteome form and function. Brief Funct Genomic Proteomic, 2006. 5(1): p. 66-73.

23. Cheng, D., et al., Relative and absolute quantification of postsynaptic density proteome isolated from rat forebrain and cerebellum. Mol Cell Proteomics, 2006. 5(6): p. 1158-70.

24. Dosemeci, A., et al., Preparation of postsynaptic density fraction from hippocampal slices and proteomic analysis. Biochem Biophys Res Commun, 2006. 339(2): p. 687-94.

25. Dosemeci, A., et al., Composition of the synaptic PSD-95 complex. Mol Cell Proteomics, 2007. 6(10): p. 1749-60.

26. Fernandez, E., et al., Targeted tandem affinity purification of PSD-95 recovers core postsynaptic complexes and schizophrenia susceptibility proteins. Mol Syst Biol, 2009. 5: p. 269.

27. Satoh, K., et al., Identification of activity-regulated proteins in the postsynaptic density fraction. Genes Cells, 2002. 7(2): p. 187-97.

28. Selimi, F., et al., Proteomic studies of a single CNS synapse type: the parallel fiber/purkinje cell synapse. PLoS Biol, 2009. 7(4): p. e83.

29. Farr, C.D., et al., Proteomic analysis of native metabotropic glutamate receptor 5 protein complexes reveals novel molecular constituents. J Neurochem, 2004. 91(2): p. 438-50.

30. Uezu, A., et al., Identification of an elaborate complex mediating postsynaptic inhibition. Science, 2016. 353(6304): p. 1123-9.

31. Frank, R.A., et al., NMDA receptors are selectively partitioned into complexes and supercomplexes during synapse maturation. Nat Commun, 2016. 7: p. 11264.

32. Schwenk, J., et al., High-resolution proteomics unravel architecture and molecular diversity of native AMPA receptor complexes. Neuron, 2012. 74(4): p. 62133.

33. Focking, M., et al., Proteomic analysis of the postsynaptic density implicates synaptic function and energy pathways in bipolar disorder. Transl Psychiatry, 2016. 6(11): p. e959.

34. Fernandez, E., et al., Arc Requires PSD95 for Assembly into Postsynaptic Complexes Involved with Neural Dysfunction and Intelligence. Cell Rep, 2017. 21(3): p. 679-691.

35. Walikonis, R.S., et al., Identification of proteins in the postsynaptic density fraction by mass spectrometry. J Neurosci, 2000. 20(11): p. 4069-80.

36. Li, J., et al., Long-term potentiation modulates synaptic phosphorylation networks and reshapes the structure of the postsynaptic interactome. Sci Signal, 2016. 9(440): p. rs8.

37. Li, J., et al., Spatiotemporal profile of postsynaptic interactomes integrates components of complex brain disorders. Nat Neurosci, 2017. 20(8): p. 1150-1161. 38. Roy, M., et al., Regional Diversity in the Postsynaptic Proteome of the Mouse Brain. Proteomes, 2018. 6(3).

39. Roy, M., et al., Proteomic analysis of postsynaptic proteins in regions of the human neocortex. Nat Neurosci, 2018. 21(1): p. 130-138.

40. Abul-Husn, N.S., et al., Systems approach to explore components and interactions in the presynapse. Proteomics, 2009. 9(12): p. 3303-15. 
41. Abul-Husn, N.S., et al., Chronic morphine alters the presynaptic protein profile: identification of novel molecular targets using proteomics and network analysis. PLoS One, 2011. 6(10): p. e25535.

42. Blondeau, F., et al., Tandem MS analysis of brain clathrin-coated vesicles reveals their critical involvement in synaptic vesicle recycling. Proc Natl Acad Sci U S A, 2004. 101(11): p. 3833-8.

43. Phillips, G.R., et al., Proteomic comparison of two fractions derived from the transsynaptic scaffold. J Neurosci Res, 2005. 81(6): p. 762-75.

44. Coughenour, H.D., R.S. Spaulding, and C.M. Thompson, The synaptic vesicle proteome: a comparative study in membrane protein identification. Proteomics, 2004. 4(10): p. 3141-55.

45. Gronborg, M., et al., Quantitative comparison of glutamatergic and GABAergic synaptic vesicles unveils selectivity for few proteins including MAL2, a novel synaptic vesicle protein. J Neurosci, 2010. 30(1): p. 2-12.

46. Takamori, S., et al., Molecular anatomy of a trafficking organelle. Cell, 2006. 127(4): p. 831-46.

47. Wilhelm, B.G., et al., Composition of isolated synaptic boutons reveals the amounts of vesicle trafficking proteins. Science, 2014. 344(6187): p. 1023-8.

48. Kokotos, A.C., et al., Activity-dependent bulk endocytosis proteome reveals a key presynaptic role for the monomeric GTPase Rab11. Proc Natl Acad Sci U S A, 2018. 115(43): p. E10177-e10186.

49. Khanna, R., A. Zougman, and E.F. Stanley, A proteomic screen for presynaptic terminal $\mathrm{N}$-type calcium channel (CaV2.2) binding partners. J Biochem Mol Biol, 2007. 40(3): p. 302-14.

50. Brinkmalm, A., et al., Targeting synaptic pathology with a novel affinity mass spectrometry approach. Mol Cell Proteomics, 2014. 13(10): p. 2584-92.

51. Gorini, G., et al., Dynamin-1 co-associates with native mouse brain BKCa channels: proteomics analysis of synaptic protein complexes. FEBS Lett, 2010. 584(5): p. 845-51.

52. Filiou, M.D., et al., Profiling of mouse synaptosome proteome and phosphoproteome by IEF. Electrophoresis, 2010. 31(8): p. 1294-301.

53. Dahlhaus, M., et al., The synaptic proteome during development and plasticity of the mouse visual cortex. Mol Cell Proteomics, 2011. 10(5): p. M110.005413.

54. Cohen, L.D., et al., Metabolic turnover of synaptic proteins: kinetics, interdependencies and implications for synaptic maintenance. PLoS One, 2013. 8(5): p. e63191.

55. Biesemann, C., et al., Proteomic screening of glutamatergic mouse brain synaptosomes isolated by fluorescence activated sorting. Embo j, 2014. 33(2): p. 15770.

56. Chang, R.Y., et al., SWATH analysis of the synaptic proteome in Alzheimer's disease. Neurochem Int, 2015. 87: p. 1-12.

57. Gonzalez-Lozano, M.A., et al., Dynamics of the mouse brain cortical synaptic proteome during postnatal brain development. Sci Rep, 2016. 6: p. 35456. 58. Heo, S., et al., Identification of long-lived synaptic proteins by proteomic analysis of synaptosome protein turnover. Proc Natl Acad Sci U S A, 2018. 115(16): p. E3827-e3836.

59. Liu, X.A., et al., New approach to capture and characterize synaptic proteome. Proc Natl Acad Sci U S A, 2014. 111(45): p. 16154-9. 
60. Alfieri, A., et al., Synaptic Interactome Mining Reveals p140Cap as a New Hub for PSD Proteins Involved in Psychiatric and Neurological Disorders. Front Mol Neurosci, 2017. 10: p. 212.

61. Kohansal-Nodehi, M., et al., Analysis of protein phosphorylation in nerve terminal reveals extensive changes in active zone proteins upon exocytosis. Elife, 2016. 5.

62. Oughtred, R., et al., The BioGRID interaction database: 2019 update. Nucleic Acids Res, 2019. 47(D1): p. D529-d541.

63. Kerrien, S., et al., The IntAct molecular interaction database in 2012. Nucleic Acids Res, 2012. 40(Database issue): p. D841-6.

64. Salwinski, L., et al., The Database of Interacting Proteins: 2004 update. Nucleic Acids Res, 2004. 32(Database issue): p. D449-51.

65. Schriml, L.M., et al., Human Disease Ontology 2018 update: classification, content and workflow expansion. Nucleic Acids Res, 2019. 47(D1): p. D955-d962. 66. Gustavsen, J.A., et al., RCy3: Network biology using Cytoscape from within $R$. F1000Res, 2019. 8: p. 1774.

67. Kang, W., et al., Identifying influential nodes in complex network based on weighted semi-local centrality, in 2016 2nd IEEE International Conference on Computer and Communications (ICCC). 2016, IEEE: Chengdu, China.

68. Nepusz, T., H. Yu, and A. Paccanaro, Detecting overlapping protein complexes in protein-protein interaction networks. Nat Methods, 2012. 9(5): p. 471-2. 69. Najafi, M., et al., Overlapping communities reveal rich structure in largescale brain networks during rest and task conditions. Neuroimage, 2016. 135: p. 92106.

70. Guimerà, R. and L.A. Nunes Amaral, Functional cartography of complex metabolic networks. Nature, 2005. 433(7028): p. 895-900.

71. McLean, C., et al., mproved Functional Enrichment Analysis of Biological Networks using Scalable Modularity Based Clustering. Journal of Proteomics and Bioinformatics, 2016. 9(1): p. 9-18.

72. Menche, J., et al., Disease networks. Uncovering disease-disease relationships through the incomplete interactome. Science, 2015. 347(6224): p. 1257601.

73. Raz, L., J. Knoefel, and K. Bhaskar, The neuropathology and cerebrovascular mechanisms of dementia. J Cereb Blood Flow Metab, 2016. 36(1): p. 172-86.

74. Hou, L., et al., Hypertension and Diagnosis of Parkinson's Disease: A MetaAnalysis of Cohort Studies. Front Neurol, 2018. 9: p. 162.

75. McGrath, E.R., et al., Blood pressure from mid- to late life and risk of incident dementia. Neurology, 2017. 89(24): p. 2447-2454.

76. Amare, A.T., et al., The genetic overlap between mood disorders and cardiometabolic diseases: a systematic review of genome wide and candidate gene studies. Transl Psychiatry, 2017. 7(1): p. e1007.

77. Gouvea, E.S., et al., Gene expression alterations related to mania and psychosis in peripheral blood of patients with a first episode of psychosis. Transl Psychiatry, 2016. 6(10): p. e908.

78. Seo, T.K. and J.L. Thorne, Information Criteria for Comparing Partition Schemes. Syst Biol, 2018. 67(4): p. 616-632.

79. Grant, S.G., Synaptopathies: diseases of the synaptome. Curr Opin Neurobiol, 2012. 22(3): p. 522-9.

80. Storey, J.D. and R. Tibshirani, Statistical significance for genomewide studies. Proc Natl Acad Sci U S A, 2003. 100(16): p. 9440-5. 